\title{
Droplet Rolling Dynamics over Hydrophobic Surface with Minute Width Channel
}

\author{
Abba Abdulhamid Abubakar ${ }^{1} \quad$ Bekir Sami Yilbas ${ }^{1,2,3,},{ }^{*}, \quad$ Hussain Al-Qahtani ${ }^{1}$ \\ Anwaruddin Siddiqui Mohammed ${ }^{1}$
}

${ }^{1}$ Mechanical Engineering Department, King Fahd University of Petroleum and Minerals (KFUPM), Dhahran 31261, Saudi Arabia.

${ }^{2}$ Center of Research Excellence in Renewable Energy (CoRE-RE), King Fahd University of Petroleum and Minerals (KFUPM), Dhahran 31261, Saudi Arabia.

${ }^{3}$ K.A.CARE Energy Research \& Innovation Center, Dhahran 31261, Saudi Arabia.

*Correspondence: bsyilbas@kfupm.edu.sa; Tel.: +966-3-860-4481

The supplementation information contains:

Number of pages $=12$

Number of figures $=5$

Number of tables $=1$

Table of contents

Page

S1. Analysis of Droplet Rolling over Channeled Hydrophobic Surface

S2. Grid Adopted in the Simulations

S3. Mesh Independent Study

S8

S4. 3D Velocity Field Developed for Droplet Rolling on Channeled and Flat Surfaces

S9

S5. Fluid Properties Adopted in the Simulations

S6. Droplet Kinetic Loss Ratio

S7. References

S12 


\section{S1. Analysis of Droplet Rolling over Channeled Hydrophobic Surface}

As the droplet wobbles, the droplet center of mass is lowered by a distance of $\chi$ (Fig. S1.a). This results in an increased solid-liquid contact radius $\left(r_{w}\right)$ and wetting area $\left(A_{w}\right)$ (Fig. S1.c). The displacement of the center of mass case of droplet rolling down an inclined plane hydrophobic surface becomes $\chi \sim \frac{r_{w}^{2}}{R}$, where $R$ is the droplet initial radius. ${ }^{1}$ The increase in solid-liquid contact area $\left(\Delta A_{w}\right)$ becomes the sum of the increase in surface area due to the surface area of the spherical cap $\left(\Delta A_{w 1}\right)$ and the the transfer of the cap volume to the the droplet volume $\left(\Delta A_{w 2}\right) .{ }^{1}$ Hence, it yields:

$\Delta A_{w} \sim r_{w}^{2}(1-\cos \theta)+R \Delta R \sim \frac{r_{w}^{4}}{R^{2}}$

here $2 \theta$ is the angle subtended by the disc at the center of the droplet and $\theta \sim \frac{l_{w}}{2 R}$.

a)

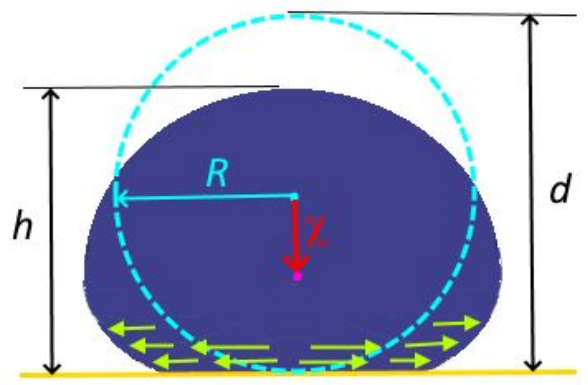

b)

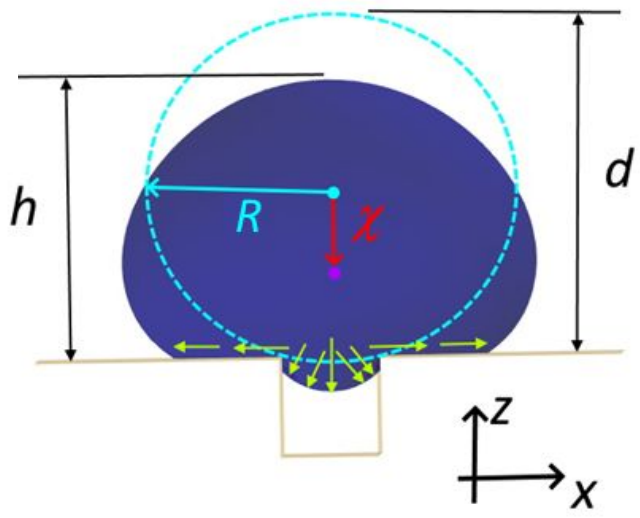

c)

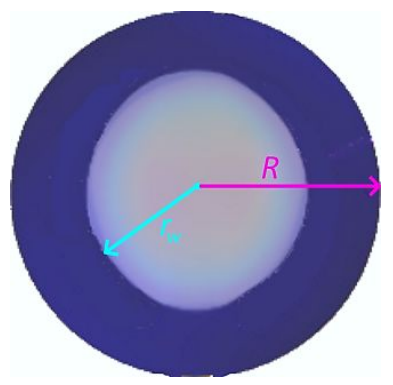

d)

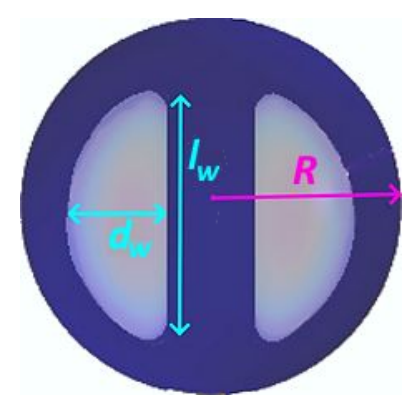

Figure S1. Geometric arrangement of droplet on plane hydrophobic surface (a) and (c). Settling of droplet on wedge channel (b) and (d). Droplet center of mass has been lowered by $\chi$.

By equating the loss in potential energy to the increase in surface energy, it becomes: 


$$
\rho g R^{3} \chi \sim \frac{\sigma r_{w}^{4}}{R^{2}}
$$

Hence, the relation for the droplet wobbling displacement and the wetting radius becomes:

$\chi \sim \frac{\rho g R^{3}}{\sigma}=B o \cdot R, \quad r_{w} \sim\left(\frac{\rho g}{\sigma}\right)^{\frac{1}{2}} R^{2}=B o^{\frac{1}{2}} \cdot R$

On the other hand, for the case of droplet rolling down an inclined hydrophobic square-wedge channel (Fig. S1.b), the wobbling displacement becomes $\chi \sim \frac{l_{w} d_{w}}{4 R}$, where $l_{w}$ is the maximum wetting length and $d_{w}$ is the maximum wetting width. The increase in the solid-liquid contact area becomes:

$\Delta A_{w} \sim\left(\frac{l_{w} d_{w}}{4}(1-\cos \theta)+R \Delta R\right) \sim \frac{l_{w}^{2} d_{w}^{2}}{16 R^{2}}+\frac{l_{w} H \delta}{2 R}$

where $\theta \sim \frac{d_{w}+\frac{H}{2}}{2 R}$ is the angle subtended by either two of wetting areas with the droplet center, $H_{S}$ is the wedge (gap) spacing and $\delta$ is the inflection height developed in the channel wedge (below the droplet wetting region). The relation for the wobbling displacement and wetting length becomes:

$\chi \sim \frac{\rho g R^{3}}{\gamma}-\delta=B o \cdot R-\delta, d_{w} \sim R-H, l_{w} \sim \frac{4 R(B o \cdot R-\delta)}{R-H}$

Here, $\gamma$ corresponds to the surface tension of the droplet fluid, $\rho$ is the fluid density, and $g$ is the acceleration due gravity.

Therefore, Eqs. S3 and S5 show that the droplet size becomes very important for rolling and sliding action down the inclined surface. The droplet rolls like a spherical marble with less wobbling when the droplet diameter becomes smaller than the capillary length, $k^{-1}=\sqrt{\frac{\gamma}{\rho g}}$, here $k^{-1}$ represents the capillarity length. Furthermore, Eq. S5 shows that the puddling displacement becomes less for 
rolling droplets in a wedge channel because of the initial inflection height $(\delta)$. In addition, the puddling displacement becomes less with increasing wedge spacing and decreasing droplet size.

Due to the large adhesion forces resulting from friction and surface roughness, the droplet sliding is inhibited during its motion down the plane. Higher adhesion force results in more rolling action for the droplet and vice versa. As a result, the difference between the advancing and receding angles becomes large. Hence, the formulation for the droplet acceleration can be derived from the balance of forces as expressed as: ${ }^{2}$

$m g \sin \alpha-F_{a d}-F_{s}-F_{f}-F_{a}=\frac{2}{5} m R \omega^{2}$

Here: $m$ is the mass of droplet, $F_{a d}$ is the adhesion force, $F_{s}$ is the shear force, $F_{f}$ is the frictional force, $F_{a}$ is the air drag force, $R$ is the droplet radius and $\omega$ is the angle of rotation.

The droplet adhesion force is proportional to the droplet fluid surface tension, contact length scale and the difference between the cosines of the advancing and receding contact angles. Hence, the adhesion force can be expressed as: ${ }^{2}$

$F_{a d}=\frac{48}{\pi^{3}} \gamma f D_{H}\left(\cos \theta_{R}-\cos \theta_{A}\right)$

Here: $\gamma$ is the droplet fluid surface tension, $f$ is solid fraction at the wetted area, $D_{H}$ is the droplet hydraulic diameter, $\theta_{A}$ is the advancing contact angle, and $\theta_{R}$ is the receding contact.

The shear force developed between the droplet liquid and the hydrophobic surface can be expressed as:

$F_{S}=A_{w}\left(\mu \frac{d V_{f}}{d z}\right)$

Eq. (S8) 
Here, $A_{w}=2 \pi l_{w} d_{w}$ is contact area, $\mu$ is dynamic viscosity, $V_{f}$ is the flow velocity inside the droplet and $z$ is the distance normal to the contact area.

The frictional force between the droplet and the hydrophobic surface can equally be expressed as:

$F_{f}=\mu_{f} m g \cos \alpha$

Here, $\mu_{f}$ is the coefficient of dynamic friction and $N=m g \cos \alpha$ is the normal reaction force.

The air drag force can be expressed as:

$F_{D}=\frac{1}{2} C_{D} \rho_{a} A_{c} v_{T}^{2}$

Here, $C_{D}$ is the drag's coefficient, $\rho_{a}$ is the air density, $A_{c}$ is the air-droplet contact area and $v_{T}$ is the droplet velocity relative to air.

By inserting the individual force terms in Eq. (S8), the droplet angular velocity can be expressed as:

$\omega=\sqrt{\frac{\frac{5}{2 m R}\left(m g \sin \alpha-\left(\frac{48}{\pi^{3}} \gamma f l_{w}\left(\cos \theta_{R}-\cos \theta_{A}\right)-A_{w}\left(\mu \frac{d V_{f}}{d z}\right)-\mu_{f} \mathrm{mg} \cos \alpha\right)\right)}{1+\frac{5}{4 m} C_{D} \rho_{a} A_{c} R}}$

Eq. $S(11)$

The droplet translational velocity can be predicted from the energy balance equation:

$E_{\text {Total }}-E_{\text {Losses }}=E_{\text {Kinetic }}$

Eq. (S12) 
Here, $E_{\text {Total }}$ is the total potential energy of the droplet at any location; $E_{\text {Drag }}$ is the energy dissipation because of frictional and other resisting forces; $E_{\text {Kinetic }}$ is the droplet kinetic energy.

Hence, the droplet translational velocity can be expressed as:

$U=\sqrt{\begin{array}{c}2 g\left[L_{T} \sin \alpha-\mu_{f} L_{T} \cos \alpha-\frac{148}{m g_{\pi}^{3}} \gamma f l_{w}\left(\cos \theta_{R}-\cos \theta_{A}\right)\right. \\ \left.-\frac{4 \gamma}{\rho g L_{T}}\left(\frac{D_{h 1}-D_{h 2}}{D_{h 1} D_{h 2}}\right)-\frac{A_{c}}{m g}\left(\mu \frac{d u_{f}}{d y}\right) L_{T}-\frac{1}{2 g} C_{D} v_{T}^{2}\right]\end{array}}$

Here, $L_{T}=\frac{h_{T}(\chi, \delta, H, R, y)}{\sin \alpha}$ is the distance between droplet center of mass and end of inclined plane, $h_{T}$ $(\chi, \delta, H, R, y)$ is the total height of the droplet center of mass with respect to the hydrophobic surface, $\alpha$ is the inclination angle, $D_{h}$ is the droplet hydraulic diameter and $m$ is the droplet mass. $h_{T}(\chi, \delta, H, R, y)$ changes with the wobbling displacement, wedge spacing, inflection height and droplet radius. 
S2. Grid Adopted in the Simulations
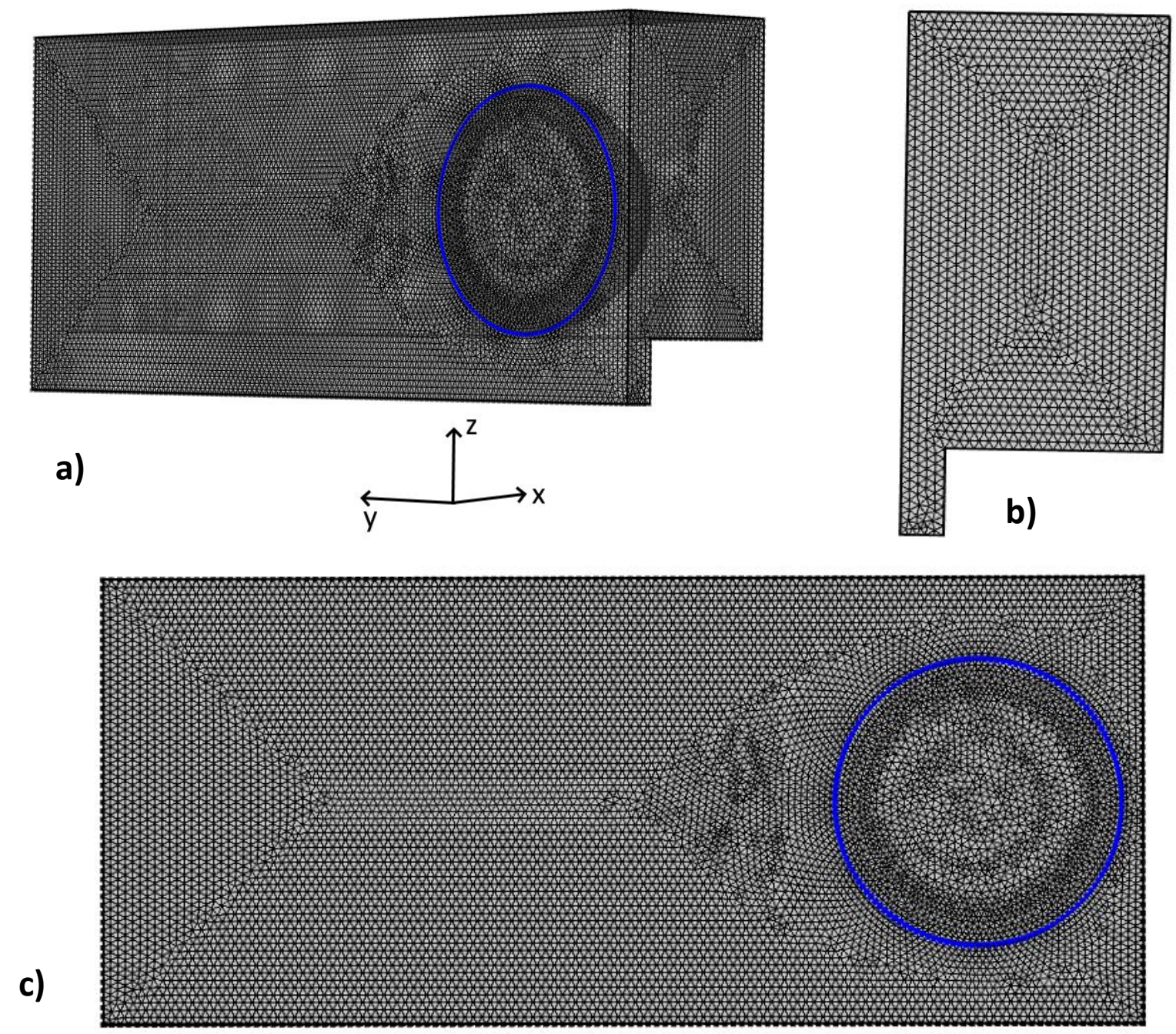

Figure S2. Grid used in the simulations: a) three-dimensional view b) cross sectional view and c) side view. Finer and dense meshes were located at the water-air and water-solid surface interfaces. 
S3. Mesh Independent Study
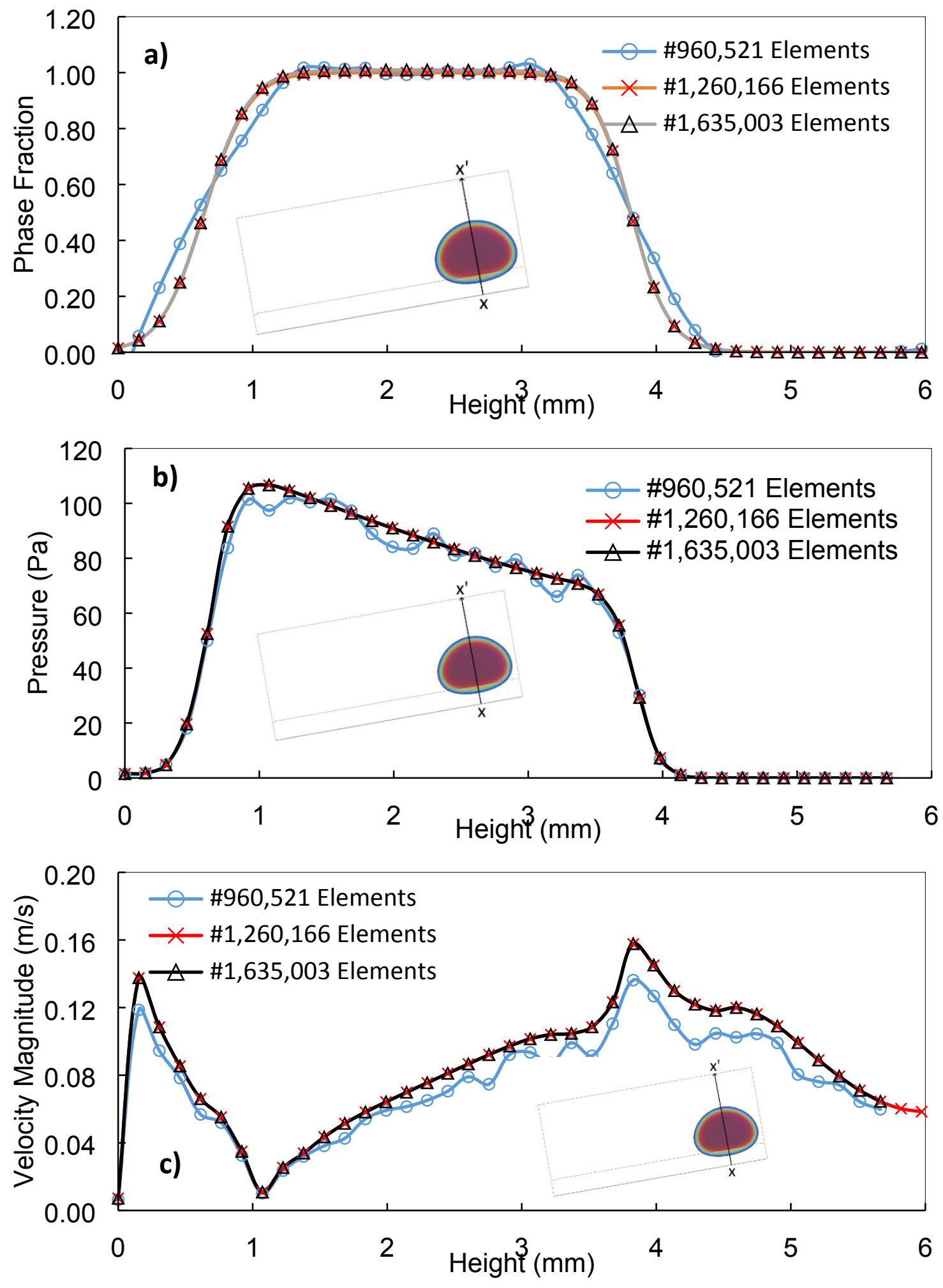
Figure S3. Grid independence study for (a) phase fraction, (b) pressure variation and (c) velocity magnitude along the droplet height from bottom to top at $0.015 \mathrm{~s}$.

\section{S4. 3D Velocity Field Developed for Droplet Rolling on Channeled and Flat Surfaces}

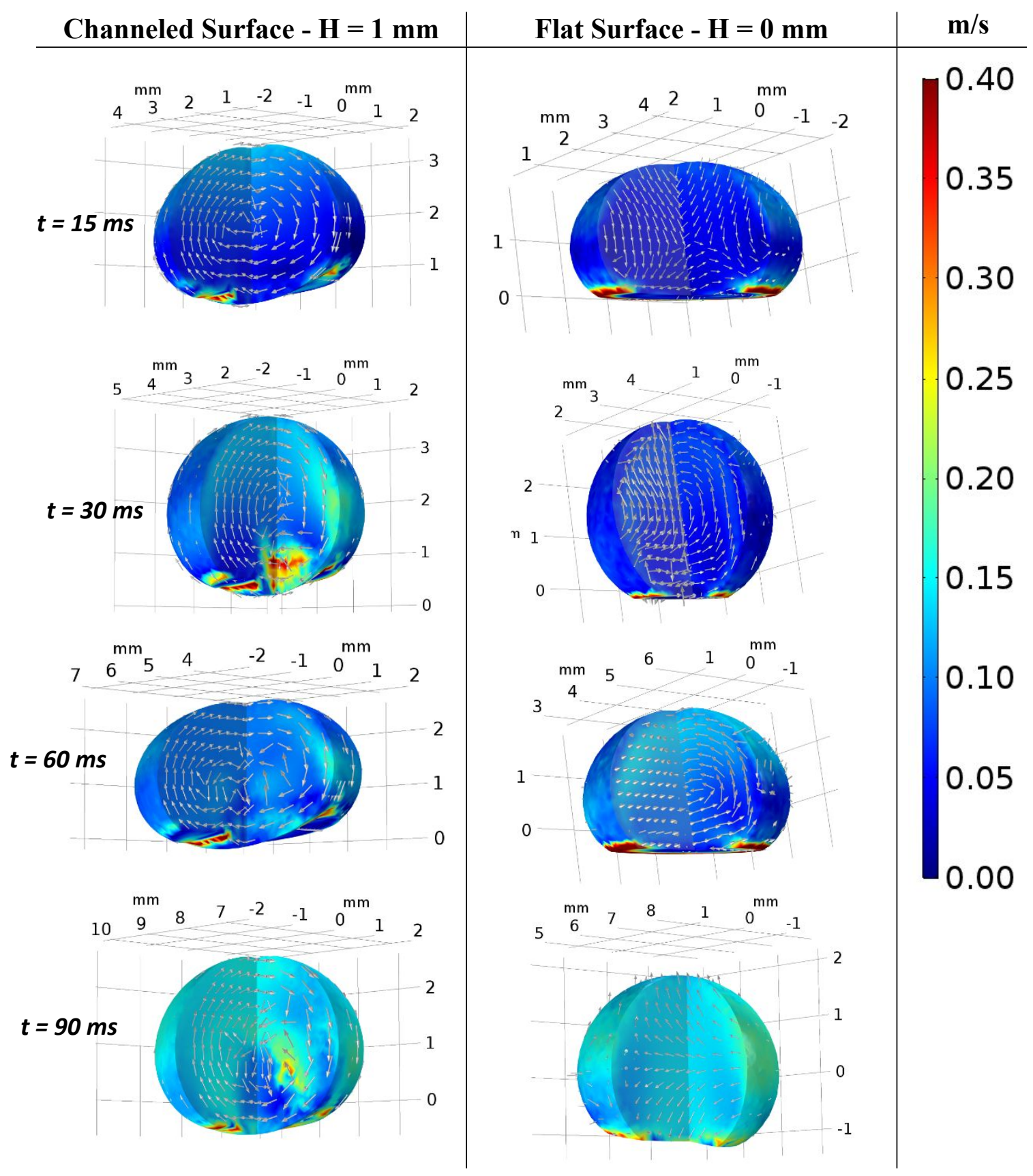

Figure S4. Velocity contours inside droplet rolling on channeled and flat hydrophobic surfaces. Droplet volume is $30 \mu \mathrm{L}$ and inclination angle is $10^{\circ}$. 


\section{S5. Fluid Properties Adopted in the Simulations}

Table S1. Fluid properties adopted in the 3D simulations

\begin{tabular}{|c|c|c|c|}
\hline property & Name & Air & Water \\
\hline$\rho\left(\mathrm{kg} / \mathrm{m}^{3}\right)$ & Density & $\begin{array}{c}6 \times 10^{-6} T^{2}-0.0036 T \\
+2.1483\end{array}$ & $\begin{array}{c}838.5+1.4 T- \\
0.003 T^{2}+3.7 \times 10^{-} \\
{ }^{7} T^{3}\end{array}$ \\
\hline$\mu(P a \cdot s)$ & Dynamic viscosity & $\begin{array}{c}1.77 \times 10^{-8} T+12.536 \\
\times 10^{-6}\end{array}$ & $\begin{array}{c}1.38-0.021 T+ \\
1.36 \times 10^{-4} T^{2}-4.65 \\
\times 10^{-7} T^{3}\end{array}$ \\
\hline$\sigma(N / m)$ & Surface tension & - & $-0.0206 T+13.41$ \\
\hline$\gamma(\mathrm{m} / \mathrm{s})$ & $\begin{array}{l}\text { Re-initialization } \\
\text { parameter }\end{array}$ & 0.6 & 0.6 \\
\hline$\epsilon(\mu m)$ & Interfacial thickness & - & $10-50$ \\
\hline$\theta_{d}$ & Contact angle & - & $150^{\circ} \pm 5^{\circ}$ \\
\hline$C_{p}(\mathrm{~J} / \mathrm{kgK})$ & Specific heat capacity & $\begin{array}{c}0.0004 T^{2}-0.1704 T+ \\
1023\end{array}$ & $\begin{array}{c}0.0112 T^{2}-7.0516 \\
T+5294.5\end{array}$ \\
\hline$k(W / m K)$ & Thermal conductivity & $\begin{array}{c}5.75 \times 10^{-5}\left(1-2.1 \times 10^{-}\right. \\
{ }^{6} T^{2}-3.17 \times 10^{-3} \mathrm{~T}+ \\
1)\end{array}$ & $\begin{array}{c}-8.354 \times 10^{-6} \\
T^{2}+6.53 \times 10^{-3} \mathrm{~T}- \\
0.5981\end{array}$ \\
\hline$D\left(\mathrm{~cm}^{2} / \mathrm{s}\right)$ & Diffusion coefficient & - & $0.0018 T-0.2913$ \\
\hline
\end{tabular}




\section{S6. Droplet Kinetic Loss Ratio}

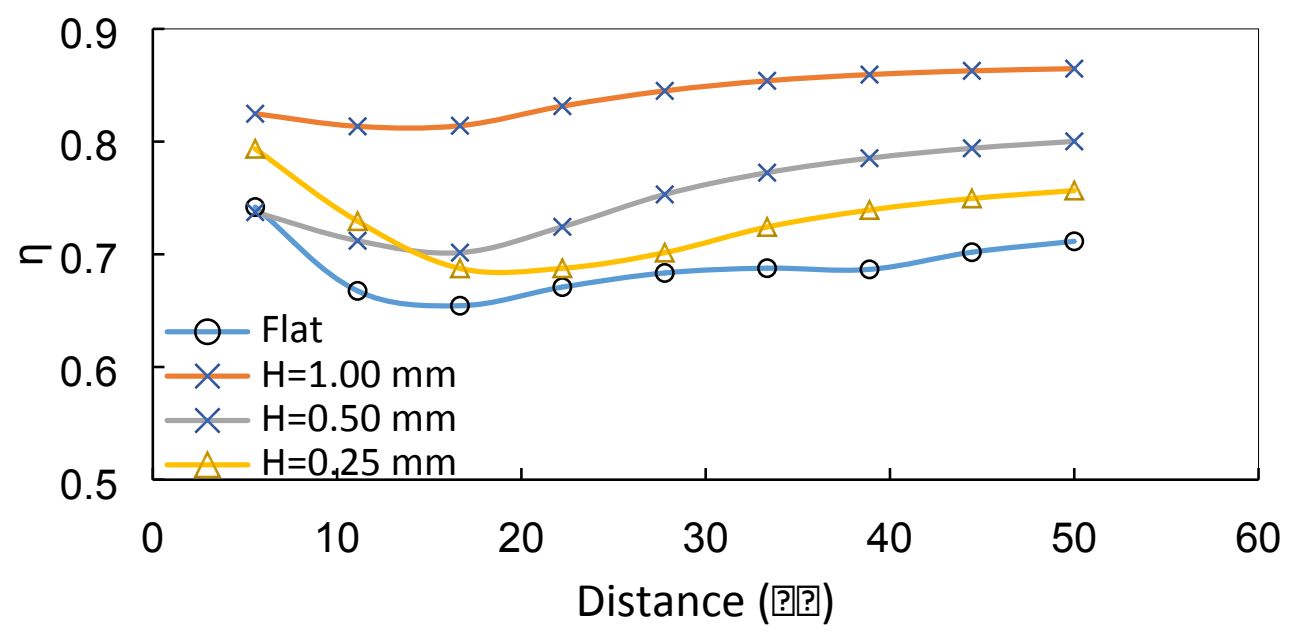

Figure S5. Droplet kinetic loss ratio $(\eta)$ along droplet path for various channeled spacings. Droplet volume is $30 \mu \mathrm{L}$ and inclination angle is $10^{\circ}$. 


\section{S6. References}

(1) Mahadevan, L.; Pomeau, Y. Rolling Droplets. Phys. fluids 1999, 11 (9), 2449-2453.

(2) Abdelmagid, G.; Yilbas, B. S.; Al-Sharafi, A.; Al-Qahtani, H.; Al-Aqeeli, N. Water Droplet on Inclined Dusty Hydrophobic Surface: Influence of Droplet Volume on Environmental Dust Particles Removal. RSC Adv. 2019, 9 (7), 3582-3596. https://doi.org/10.1039/C8RA10092F. 